\title{
Treatment of severe mucositis pain with oral ketamine mouthwash
}

\author{
Alexandra Shillingburg ${ }^{1,2}$, Abraham S. Kanate $^{2}$, Mehdi Hamadani $^{3}$, Sijin Wen $^{4}$, Michael \\ Craig $^{2}$, and Aaron Cumpston ${ }^{1,2}$ \\ ${ }^{1}$ Department of Pharmacy, West Virginia University Medicine, P.O. Box 8045, Medical Center \\ Drive, Morgantown, WV 26506, USA \\ 2Osborn Hematopoietic Malignancy and Transplantation Program, West Virginia University \\ Cancer Institute, Morgantown, WV, USA \\ ${ }^{3}$ Division of Hematology/Oncology, Medical College of Wisconsin, Milwaukee, WI, USA \\ ${ }^{4}$ Department of Biostatistics, West Virginia University Cancer Institute, Morgantown, WV, USA
}

\begin{abstract}
Purpose-Mucositis is a significant complication of intensive chemotherapy or hematopoietic cell transplantation (HCT), with few treatment options. Ketamine mouthwashes have been used for pain relief, but supporting evidence is limited. The primary objective of this study was to assess the reduction in pain intensity of stomatodynia and odynophagia compared to baseline assessment.
\end{abstract}

Methods-This open-label, prospective, phase II interventional study (NCT01566448) was conducted from February 2012 through July 2015. Patients with grade 3 or 4 oral mucositis according to the World Health Organization (WHO) scale as a result of chemotherapy were treated with ketamine mouthwash $20 \mathrm{mg} / 5 \mathrm{~mL}$ four times daily and every $4 \mathrm{~h}$ as needed.

Results-Thirty patients were enrolled and a total of 136 assessments were conducted. A statistically significant reduction in pain scores of 2 and 3 points was achieved after $1 \mathrm{~h}$ and 3 days, respectively $(p<0.0001, p=0.0003)$. Pain scores were significantly improved while swallowing, reduced 1 and 4 points at 1-h and 3-day assessment, respectively ( $p=0.0006, p=$ 0.0001). No patients developed adverse effects related to ketamine administration.

Conclusion-Ketamine mouthwashes resulted in clinically meaningful and statistically significant reduction in pain scores, have an acceptable safety profile, and can be a useful adjunctive treatment in the multi-modal management of severe mucositis.

\section{Keywords}

Ketamine; Mouthwash; Oral; Mucositis; Pain; Transplant

Correspondence to: Alexandra Shillingburg.

Compliance with ethical standards This study (ClinicalTrials.gov Identifier, NCT01566448) was approved by the Protocol Review Monitoring Committee at the West Virginia University (WVU) Cancer Institute and the Institutional Review Board at WVU Medicine. Conflict of interest The authors declare that they have no conflict of interest. 


\section{Introduction}

Mucositis has significant quality of life and clinical consequences for patients undergoing antineoplastic cytotoxic therapies. Oral mucositis occurs in approximately $40 \%$ of patients receiving standard dose chemotherapy, $80 \%$ of patients receiving radiation therapy to the head and neck, and up to $100 \%$ of patients undergoing a hematopoietic cell transplant (HCT) [1]. Mucositis and associated pain are consistently reported as extremely distressing for patients and may involve erythema, inflammation, bleeding, and ulceration. Pain from oral mucositis has been reported as one of the most debilitating side effect by patients receiving HCT. Mucositis also has the potential to impact the effectiveness of cancer treatment as it is a dose-limiting toxicity resulting in cessation or reduction of treatment in $35 \%$ of patients receiving chemotherapy [2-4].

As a result, many agents and strategies have been investigated to alleviate this pain, to prevent the incidence of mucositis, or to decrease the severity of mucositis. Unfortunately, few available options have proven useful. One marketed agent is a recombinant human keratinocyte growth factor, palifermin, and despite its approval to reduce the incidence and duration of oral mucositis, it has seen limited utility due to questionable clinical benefit and high acquisition costs. Current standard of care focuses on palliation and includes systemic opiate analgesics for moderate to severe mucositis pain, topical anesthetics and mucosal coating agents for moderate pain, and bland rinses for mild pain. Data supporting these management options are limited. Other agents that have been investigated with variable responses are oral capsaicin, oral sulfasalazine, and growth factor mouthwashes [5].

Ketamine is a sedative hypnotic with anesthetic and analgesic properties and with reported benefit when used topically. Ketamine works by selectively depressing the thalamoneocortical system, non-competitively blocking $N$-methyl-D-aspartate (NMDA) receptors, and having intrinsic sympathomimetic activity. Ketamine also appears to selectively interrupt association pathways in the brain producing somatesthetic sensory blockade. Ketamine is FDA approved for induction and maintenance of general anesthesia but has also been used for procedural sedation, refractory severe pain, and acute respiratory failure in children. Local administration of ketamine to the limbs of patients with complex regional pain syndrome has been shown to cause a reduction in allodynia in a double-blind placebo controlled study. Peripheral administration of ketamine was shown to have antinociceptive efficacy similar to that of systemic administration, likely mediated by NMDA antagonism [6]. Ketamine also has the modest anti-inflammatory properties which could be beneficial in mucositis pain relief.

Ketamine oral rinse was described in a case report of a 32-year-old female with radiationinduced mucositis pain refractory to compounded topical solutions (containing lidocaine, diphenhydramine, nystatin, and magnesium hydroxide/aluminum hydroxide), transdermal fentanyl, and intravenous hydromorphone. She experienced decreased stomatodynia and odynophagia and was able to decrease the dose of her systemic opiate analgesics [7]. A retrospective chart review of ketamine mouthwash in eight patients over a period of 4 years was conducted but the inconsistent reporting of pain scores was difficult to quantify. However, the authors noted that ketamine may provide a viable treatment option for 
mucositis pain [8]. We prospectively evaluated ketamine as an oral mouthwash to provide pain relief for patients with severe oral mucositis.

\section{Methods}

\section{Study objectives}

The primary objective of this study was to assess the reduction in pain intensity of stomatodynia and odynophagia compared to baseline assessment as reported by the patient on a numeric scale. Secondary objectives include assessing patient-reported onset and duration of effect (as defined below), reduction in both narcotic analgesic use and topical lidocaine use, and improvement in patient-reported sleep quality, safety, and tolerability.

\section{Patients}

Patients eligible for inclusion were 18 years of age or older, received at least one dose of chemotherapy, had grade 3 or 4 oral mucositis according to the World Health Organization (WHO) oral mucositis scale [9], and signed the informed consent. Causative chemotherapy could include conditioning regimens for allograft, autograft, or chemotherapy alone. Patients were excluded if they had any documented hypersensitivity to ketamine; received prior ketamine doses by any route within $48 \mathrm{~h}$ of study initiation; were pregnant or breastfeeding; had schizophrenia, acute psychosis, or any psychiatric disorder that could be dangerous if exacerbated; or were unable to render informed consent. This study (ClinicalTrials.gov Identifier, NCT01566448) was approved by the Protocol Review Monitoring Committee at the West Virginia University (WVU) Cancer Institute and the Institutional Review Board at WVU Medicine.

\section{Preparation}

The solution was prepared by the pharmacy in batches of 24 individual oral syringes containing a 5-mL dose. A 5-mL vial of ketamine $100 \mathrm{mg} / \mathrm{mL}$ solution was diluted with normal saline to a final concentration of $20 \mathrm{mg} / \mathrm{mL}$, drawn into unit-dose syringes, sealed with tamper-evident caps, and stored in automated dispensing cabinets on the patient's unit. Each syringe was given an expiration date of 7 days from preparation when stored at room temperature [10].

\section{Study protocol}

This was an open-label, prospective, phase II interventional study conducted at a single institution. Patients were treated with ketamine oral mouthwash $20 \mathrm{mg} / 5 \mathrm{~mL}$ swish and spit scheduled four times daily and additional doses permitted every $4 \mathrm{~h}$ as needed. Dosing was based on methods from prior published retrospective reports. [7, 8] Patients were asked to swish the solution for at least $30 \mathrm{~s}$. Gargling the solution was allowed but patients were asked not to swallow the dose. All patients were permitted to continue analgesic therapy and topical lidocaine-based solutions, such as compounded admixtures with any combination of viscous lidocaine, diphenhydramine, nystatin, aluminum/magnesium antacids, and/or corticosteroids, often referred to as "Clark's solution" or "magic mouthwash" [11]. Topical lidocaine doses were recommended to be separated by at least $1 \mathrm{~h}$ from the ketamine rinse. Patients were also asked to refrain from eating or drinking for at least 30 min after each 
ketamine dose. Patients were removed from the study on the day when their mucositis had resolved to less than grade 3. Removal from the study was also permitted by request from either the patient or physician or due to lack of efficacy, defined as no decrease in pain scores for three consecutive days.

\section{Assessment}

Baseline assessments were performed prior to initiating therapy with ketamine mouthwashes, $1 \mathrm{~h}$ after the first dose of ketamine and then daily thereafter for up to 7 days. Pain scores were assessed on a numeric scale from 0 to 10, with 0 representing no pain and 10 representing the worst pain. Patients were asked to score pain at rest and when swallowing. Sleep quality was assessed on a 0 to 10 numeric scale, with 0 representing no sleep and 10 representing optimal sleep. Patients were assessed for food intake by the investigators, classified as none, liquids only, soft food only, or normal diet. Patients selfreported the onset and duration of effects categorically. Onset of effect was reported as either no effect, 0-15 $\mathrm{min}, 16-30 \mathrm{~min}, 31-45 \mathrm{~min}, 46-60 \mathrm{~min}$, or greater than $1 \mathrm{~h}$. Duration of effect was reported as either no effect, less than $1 \mathrm{~h}, 1-2 \mathrm{~h}, 2-3 \mathrm{~h}, 3-4 \mathrm{~h}$, or 4 or more hours. The WHO oral mucositis severity grade was recorded daily.

Topical lidocaine-based solution usage was documented as the number of administrations per 24-h period. Narcotic analgesic use was reported as intravenous (IV) morphine equivalents over a 24-h period. All analgesics including oral, transdermal, intermittent IV, and PCA were converted to IV morphine equivalents based on a standard opioid equivalency table [12] (see Table 1). Baseline assessment of lidocaine use and morphine equivalents included the $24 \mathrm{~h}$ prior to the first dose of ketamine. Investigators assessed for adverse events, altered mental status, and recorded patient-reported outcomes (PRO) on data collection sheets with daily assessments of patient's alertness, communication responsiveness, and full orientation with their situation and surroundings. Wilcoxon's sum rank test was used in the data analysis on continuous variables and Fisher's exact test was used for categorical variables.

\section{Results}

Thirty patients were enrolled and a total of 136 unique assessments were conducted. Patients were on study for a median of 4 days (range 1-9) and received a median of 3.5 doses of ketamine per patient/day (range 1-5.7). The majority of patients had a diagnosis of acute myeloid leukemia (AML) and received myeloablative conditioning for an allogeneic transplant. The median grade of mucositis at baseline and on each study day was 3 with a range of 3-4. No patients in this study received palifermin. Baseline demographic details are described in Table 2.

A statistically significant decrease in pain scores was seen in the assessments performed both when resting and when swallowing at the 1-h assessment (reduction of 2 and 2.5 points, respectively, $p<0.0001$ and $p=0.0006$ ) and sustained at the day 3 assessment (reduction of 3 and 4 points, respectively, $p=0.0003$ and $p=0.0001$ ). Sleep quality was assessed as a surrogate marker for patient quality of life. Sleep quality significantly improved from a median rating of 5 to $6(p=0.006)$ after the first night and then sustained that improvement 
through day 3 ( $p=0.034$ ). Concomitant lidocaine-based oral solutions were used by $90 \%$ ( $n$ $=27$ ) of patients and were significantly reduced by a median of 1 dose per patient/day (range +3 to -4$)(p=0.029)$ after initiation of ketamine mouthwashes. The median daily requirement of IV morphine equivalents per patient appeared to be reduced by $22 \mathrm{mg}$ (range +85.8 to $-70.6 \mathrm{mg})$, but this did not reach statistical significance $(p=0.145)$. Patient outcomes are summarized in Table 3.

The onset of action was noted within 15 min of the dose in the majority of patients and reported to last for 1-3 h (Fig. 1). Tolerability of the solution was acceptable and several patients commented that the solution was preferable to the lidocaine-based solutions due to less burning and irritation. Twenty-nine of the 30 patients were removed from study due to resolution of their mucositis to less than grade 3. One patient (3\%) was withdrawn from the study at the physician's discretion due to altered mental status, reported to be due to continuous intravenous narcotics and onset of sepsis and not deemed related to ketamine mouthwashes.

\section{Discussion}

Our study is the first prospective analysis of topical ketamine for the reduction of mucositis pain. We found a significant reduction in pain scores from baseline, as well as an improvement in sleep quality. Although pain is a subjective measure and varies greatly from patient to patient, applying a numeric scale to rate pain has been widely adopted as an acceptable way to quantify and track changes in intensity. Previous studies evaluating mucositis pain relief have cited a reduction in 2 points on a numeric 10-point scale to be "clinically" significant $[13,14]$. While the assessment of generalized body pain has become routine in most institutions and is available in most charts for review, in order to accurately assess localized pain associated with oral mucositis and the relief of that pain by a specific intervention, a prospective evaluation of data was required.

Comparing outcomes from subsequent assessments to baseline (prior to ketamine) removed inter-patient variability of pain scores. The possibility of intervention bias (patients reporting lower pain scores as they are receiving treatment) was likely minimal, since no changes were made to the patients' current intensive systemic and local treatments at the time of ketamine intervention. Most of the patients were previously receiving topical lidocaine in the study and we did not inform patients that we would be recording the amount used in order to minimize patient request bias. One-hour assessment after initial dose was thought to be the most accurate predictor of the efficacy of ketamine mouthwashes, as the severity of mucositis will be most similar to baseline. As the time from baseline assessment increased, we acknowledge that there would be greater variation in the severity of their mucositis which may be reflected in the pain assessments.

We assessed the tolerability and palatability of the oral solution. For patients experiencing this degree of mucosal damage, formulations that cause additional discomfort are not ideal. All patients reported that the solution was tolerable, and in most cases, preferable to the lidocaine-based solutions. Some patients reported a metallic taste, but that it was not overly unpleasant. A few patients commented that the $5-\mathrm{mL}$ volume was not enough quantity and 
suggested a larger volume would be more beneficial. Absorption through mucosal membranes should be minimal, but with severe ulcerations, the true amount of systemic absorption is unknown. Ketamine is known to occasionally produce laryngospasm which could be problematic in a patient with mucositis, but no incidences of this occurred during the study. No patients were noted to have systemic toxicities, specifically psychogenic or dissociative effects, related to mucosal absorption of ketamine.

Given that our study did not find high rates of adverse effects, consideration could be given to the investigation of higher concentrations of ketamine or larger volumes in attempt to increase the topical efficacy. In future investigations, although patients had clinical assessments performed as part of their standard of care, detailed assessments for ketaminespecific adverse effects would be beneficial, as the authors acknowledge that patientreported outcomes may be subject to ketamine-induced amnesia. Other modifications that may warrant investigation would be substituting the normal saline diluent for a more viscous substance to allow for mucosal adhesion or potentially adding flavoring for palatability. Additional studies evaluating ketamine and lidocaine on an alternating schedule or possibly in a combination product may also show additional pain relief. A recent large phase III randomized study found that a doxepin rinse was effective at reducing pain scores by a mean of 2 (on a 10-point scale) which is synonymous with the results we report in this study with ketamine rinses; an evaluation directly comparing the two or the combination of both may give further information to help manage the pain of oral mucositis.

\section{Conclusion}

In our study, oral ketamine mouthwash use resulted in a clinically meaningful and statistically significant reduction in pain scores for stomatodynia and odynophagia, improved sleep quality, and reduced oral lidocaine utilization for patients with severe oral mucositis. There was no apparent toxicity, oral tolerability was favorable, and cost was minimal. Ketamine mouthwash could provide a useful adjunctive treatment in the multimodal management of severe oral mucositis associated with chemotherapy.

\section{References}

1. Harris DJ. Cancer treatment-induced mucositis pain: strategies for assessment and management. Ther Clin Risk Manag. 2006; 2:251-258. [PubMed: 18360600]

2. Bellam LA, Epstein JB, Rose-Ped A, Martin P, Fuchs HJ. Patient reports of complications of bone marrow transplantation. Support Care Cancer. 2000; 8:33-39. [PubMed: 10650895]

3. Sonis ST, Elting LS, Keefe D, Peterson DE, Schubert M, Hauer-Jensen M, et al. Perspectives on cancer therapy-induced mucosal injury: pathogenesis, measurement, epidemiology, and consequences for patients. Cancer. 2004; 100:1995-2025. [PubMed: 15108222]

4. Borbasi S, Cameron K, Quested B, Olver I, To B, Evans D. More than a sore mouth: patients' experience of oral mucositis. Oncol Nurs Forum. 2002; 29:1051-1057. [PubMed: 12183754]

5. Network NCC. NCCN Task Force Report: prevention and management of mucositis in cancer care. J Natl Compr Cancer Netw. 2008; 1:S1-21.

6. Finch PM, Knudsen L, Drummond PD. Reduction of allodynia in patients with complex regional pain syndrome: a double-blind placebo-controlled trial of topical ketamine. Pain. 2009; 146:18-25. [PubMed: 19703730]

7. Slatkin NE, Rhiner M. Topical ketamine in the treatment of mucositis pain. Pain Med. 2003; 4:298303.8. [PubMed: 12974832] 
8. Ryan AJ, Lin F, Atayee RS. Ketamine mouthwash for mucositis pain. J Palliat Med. 2009; 12:989991. [PubMed: 19708855]

9. World Health Organization. Handbook for reporting results of cancer treatment. World Health Organization; Geneva: 1979. p. 15-22.

10. Roy JJ, Hildgen P. Stability of morphine-ketamine mixtures in $0.9 \%$ sodium chloride injection packaged in syringes, plastic bags and medication cassette reservoirs. Int J Pharm Compound. 2000; 4:225-228.

11. PL Detail-Document \#301105, Magic mouthwash recipes. Pharmacist's Letter/Prescriber's Letter. 2014

12. PL Detail-Document, Equianalgesic dosing of opioids for pain management. Pharmacist's Letter/ Prescriber's Letter. 2012

13. Hadjieva T, Cavallin-Stahl E, Linden M, Tiberg F. Treatment of oral mucositis pain following radiation therapy for head-and-neck cancer using a bioadhesive barrier-forming lipid solution. Support Care Cancer. 2014; 22:1557-1562. [PubMed: 24442926]

14. Leenstra JL, Miller RC, Qin R, Martenson JA, Dornfield KJ, Bearden JD, et al. Doxepin rinse versus placebo in the treatment of acute oral mucositis pain in patients receiving head and neck radiotherapy with or without chemotherapy: a phase III, randomized, double-blind trial (NCCTGN09C6). J Clin Oncol. 2014; 32:1571-1577. [PubMed: 24733799] 


\begin{tabular}{|c|c|c|c|}
\hline \multicolumn{4}{|l|}{$\mathrm{a}$} \\
\hline$>1 \mathrm{hr}$ & \multicolumn{3}{|l|}{0} \\
\hline $45-60 \mathrm{~min}$ & \multicolumn{3}{|l|}{0} \\
\hline $30-45 \mathrm{~min}$ & \multicolumn{3}{|l|}{0} \\
\hline $15-30 \mathrm{~min}$ & \multicolumn{3}{|l|}{0} \\
\hline $0-15 \mathrm{~min}$ & \multicolumn{3}{|r|}{28} \\
\hline No Effect & \multicolumn{3}{|l|}{2} \\
\hline & 10 & 20 & 30 \\
\hline & Tumber of & nts & \\
\hline
\end{tabular}

b

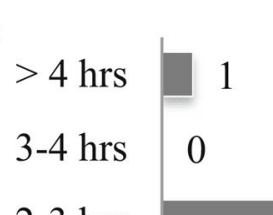

2-3 hrs

$1-2$ hrs

$<1 \mathrm{hr}$

No Effect
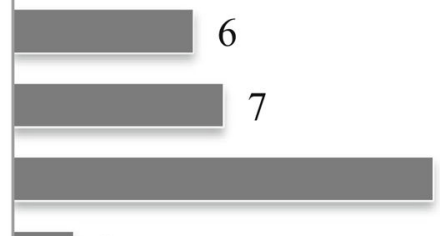

14

2

0

5

10

15

Fig. 1.

a Onset of effect from ketamine mouthwashes. b Duration of effect of ketamine mouthwashes 


\section{Table 1}

Opioid equivalency table

\begin{tabular}{lll}
\hline Drug & Dose $(\mathbf{m g})$ parenteral & Dose $(\mathbf{m g})$ oral \\
\hline Morphine & 10 & 30 \\
Hydromorphone & 1.5 & 7.5 \\
Oxycodone & & 20 \\
Methadone & 5 & 5 \\
Codeine & 120 & 200 \\
Hydrocodone & & 30 \\
Meperidine & 75 & 300 \\
Fentanyl & 0.1 & \\
\hline
\end{tabular}

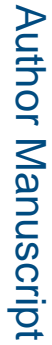

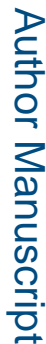

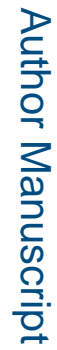

Support Care Cancer. Author manuscript; available in PMC 2018 July 01. 


\section{Table 2}

\section{Demographics}

\begin{tabular}{|c|c|c|}
\hline Number of patients & $N=\mathbf{3 0}$ & $(\%)$ \\
\hline Median age in years (range) & 44 & $(23-67)$ \\
\hline \multicolumn{3}{|l|}{ Gender } \\
\hline Female & 17 & $(57)$ \\
\hline Male & 13 & $(43)$ \\
\hline \multicolumn{3}{|l|}{ WHO mucositis grade at enrollment } \\
\hline Grade 3 & 16 & $(53)$ \\
\hline Grade 4 & 14 & $(47)$ \\
\hline \multicolumn{3}{|l|}{ Primary malignancy } \\
\hline AML & 17 & $(57)$ \\
\hline Lymphoma & 6 & $(20)$ \\
\hline CML & 2 & (7) \\
\hline ALL & 2 & (7) \\
\hline Other & 3 & $(10)$ \\
\hline llogeneic conditioning & 23 & $(77)$ \\
\hline Autologous conditioning (CBV) & 2 & (7) \\
\hline Other & 5 & (16) \\
\hline Allogeneic conditioning regimen: & $N=23$ & \\
\hline $\mathrm{Flu} / \mathrm{Bu} 4$ & 12 & $(52)$ \\
\hline $\mathrm{Bu} / \mathrm{Cy}$ & 5 & $(22)$ \\
\hline $\mathrm{Flu} / \mathrm{Bu} 4 / \mathrm{TBI}$ & 3 & (13) \\
\hline $\mathrm{Cy} / \mathrm{TBI}$ & 2 & (9) \\
\hline $\mathrm{Flu} / \mathrm{Cy} / \mathrm{TBI}$ & 1 & (4) \\
\hline Parenteral nutrition & 8 & (27) \\
\hline
\end{tabular}

$A M L$ acute myeloid leukemia, $C M L$ chronic myeloid leukemia, $A L L$ acute lymphoid leukemia, $C B V$ cyclophosphamide/carmustine/etoposide, Flu/Bu4 fludarabine/busulfan, Bu/Cy busulfan/cyclophosphamide, Flu/Bu4/TBI fludarabine/busulfan/total body irradiation, $C y / T B I$ cyclophosphamide/total body irradiation, Flu/Cy/TBI fludarabine/cyclophosphamide/total body irradiation 


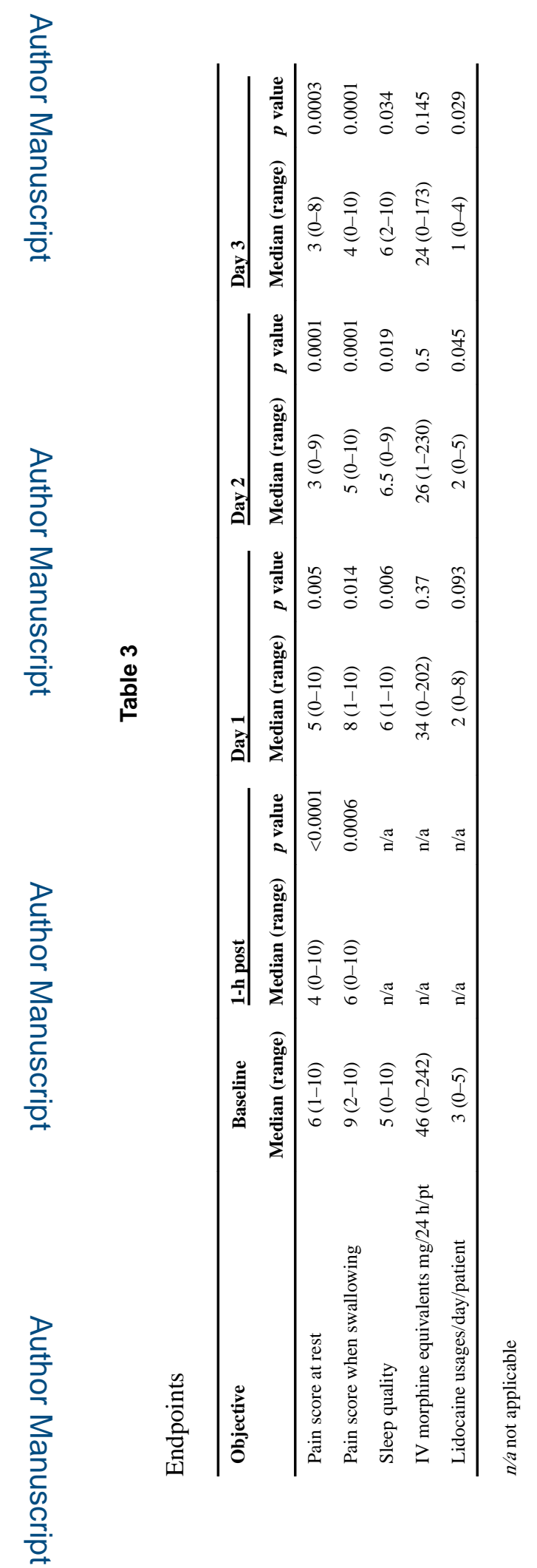

Support Care Cancer. Author manuscript; available in PMC 2018 July 01. 